\title{
Comparison of Hybrid RANS/LES Models for Rocket Combustion Chamber Simulations
}

\author{
Timo Seitz* and Peter Gerlinger ${ }^{\dagger}$ \\ Institute of Combustion Technology for Aerospace Engineering (IVLR), University of Stuttgart, 70569 Stuttgart, \\ Germany
}

\begin{abstract}
In the development process of rocket combustion chambers, the demand of high-fidelity numerical models is rising in order to capture all the occurring phenomena. As wall-resolved large eddy simulations (LES) are extremely expensive for the conditions prevailing in these combustors, hybrid Reynolds-Averaged Navier Stokes (RANS)/LES calculations are a good compromise. In this work, two hybrid RANS/LES methods, namely the so-called iDDES and $l^{2}-\omega$-DDES, are compared to each other with the aim of testing their applicability for rocket combustion chambers. These are first validated on non-reacting test cases. Although the mean quantities do not differ significantly, the instantaneous flow fields exhibit a different behavior. In addition, simulations of a laboratory-scale rocket combustion chamber are performed. The results show clear differences in the prediction of some of the mean quantities such as the wall heat flux, highlighting the strong dependence of the combustion process from the turbulence modeling.
\end{abstract}

\section{Introduction}

The accurate and reliable prediction of quantities such as the wall heat flux, the pressure fluctuations or the flow field is a key aspect in the design and development process of rocket combustion chambers. In high-pressure environments, experiments, although being the most credible approach, are often restricted to single measurable quantities. In contrast, numerical simulations provide extensive data sets.

With increasing computational resources in recent years, three-dimensional and time-resolved rocket combustion chamber simulations became more and more attractive. However, it is still not possible to perform a simulation of a complete combustion chamber with direct numerical simulation (DNS) that resolves all turbulent scales. One limiting factor is the grid requirement in the boundary layer which scales with the Reynolds number [1]. Likewise, wall-resolved large eddy simulations (LES) are extremely costly and tedious. Although the computational requirements of a LES are smaller than for DNS, the scaling of the effort is similar [2]. In contrast to these methods, (unsteady) Reynolds-averaged Navier-Stokes (RANS) simulations are computationally less demanding, but almost no turbulent content is resolved. Therefore, a good tradeoff between computational cost and accuracy are so-called hybrid RANS/LES methods. The basic idea of these approaches is to use an established RANS model in the boundary layer and switching to LES mode in free shear flows. Several strategies exist for combining RANS and LES models. One of the most popular approaches originates from Spalart et al. [3] and is called detached-eddy simulation (DES). This method is based on the Spalart-Allmaras turbulence model. The main idea of this approach is to introduce a length scale $l_{\mathrm{DES}}$ that forces the model to operate in both the RANS and the LES mode depending on its value. This avoids the high computational costs that would be inherent to wall-resolved LES while at the same time taking advantage of LES in the core flow. Strelets [4] extended this version to two-equation turbulence models by modifying the dissipation term of the transport equation for the turbulent kinetic energy $k$. Various drawbacks, including artificial grid induced separation (GIS) or log-layer mismatch (LLM), are known. Hence, a couple of models have emerged from these original formulations. In order to overcome GIS, Spalart et al. [5] introduced a shielding function to switch between RANS and LES mode. This yields the delayed DES (DDES). The introduction of a revised length scale and the inclusion of additional blending functions reduces the LLM. This formulation is termed improved DDES (iDDES) [6] and is one of the models considered in this work. Reddy et al. [7] used the length scale to alter the production term of the $k$-equation thus forming a Smagorinsky-type LES model. In addition, they also used an adjusted length scale in order to avoid LLM. This method is called $l^{2}-\omega$-DDES. The procedure of $l^{2}-\omega$-DDES facilitates an dynamic approach [8]. In this work, the $l^{2}-\omega$-DDES formulation of Reddy et al. [7] is used as second applied hybrid RANS/LES method. Recent hybrid RANS/LES methods

\footnotetext{
*PhD student, timo.seitz@dlr.de

†Professor, peter.gerlinger@dlr.de
} 
combine elements of various other approaches. For example, the production-limited DDES of Ding et al. [9] uses the concept of the $l^{2}-\omega$-DDES combined with empirical functions occurring in the iDDES plus a blending of the turbulent viscosity.

In this work, the two mentioned DDES models are compared to each other in order to test their applicability for rocket combustion chamber simulations. A first validation on non-reacting test cases allows identifying their general behavior. The obtained results also provide the basis for a better understanding of the outcome of rocket combustion chamber simulations as a different description of the turbulence affects the combustion. Wall heat flux measurements of the so-called PennState combustor are then used to benchmark the different simulations.

The remainder of this work is structured as follows. In the subsequent section, the used numerical method is described. Special attention is paid to the applied hybrid RANS/LES methods. These are characterized in Sec. III]. Before presenting results of a rocket combustion chamber in Sec. $\mathrm{V}$ ] the Sec. IV]discusses the behavior of the applied methods for non-reactive flows. Finally, Sec. VI gives a summary.

\section{Numerical Method}

All simulations are performed with the in-house code TASCOM3D (Turbulent All Speed Combustion Multigrid 3D). Over the past twenty years, TASCOM3D has been developed and successfully applied to a wide range of reacting and non-reacting flows in both the subsonic and the supersonic regime e.g [10-12]. The following subsections shortly describe the governing equations and the applied numerical methods.

\section{A. Governing Equations}

Turbulent flow and combustion processes are described by the compressible Navier-Stokes equations that, in addition to the conservation of mass, momentum and energy, include equations for turbulence modeling and species transport. The set of governing equations is given by

$$
\frac{\partial \mathbf{Q}}{\partial t}+\frac{\partial\left(\mathbf{F}-\mathbf{F}_{v}\right)}{\partial x}+\frac{\partial\left(\mathbf{G}-\mathbf{G}_{v}\right)}{\partial y}+\frac{\partial\left(\mathbf{H}-\mathbf{H}_{v}\right)}{\partial z}=\mathbf{S}
$$

with the vector of conservative quantities

$$
\mathbf{Q}=\left[\bar{\rho}, \bar{\rho} \tilde{u}, \bar{\rho} \tilde{v}, \bar{\rho} \tilde{w}, \bar{\rho} \tilde{E}, \bar{\rho} k, \bar{\rho} \omega, \bar{\rho} \sigma_{T}, \bar{\rho} \sigma_{Y}, \bar{\rho} \tilde{Y}_{i}\right]^{\top}, i=1, \ldots, N_{k}-1 .
$$

Here, the vector $\mathbf{Q}$ consists of the Reynolds-averaged density $\bar{\rho}$, the Favre-averaged velocities $\tilde{u}, \tilde{v}, \tilde{w}$ in each direction, the total specific energy $\tilde{E}$, the turbulence quantities $k$ and $\omega$, the temperature variance $\sigma_{T}$, the sum of the variances of all species mass fractions $\sigma_{Y}$ as well as the $N_{k}-1$ independent species mass fractions $\tilde{Y}_{i} . N_{k}$ is the number of considered species. For readability, the overbars $(\bar{\cdot})$ respectively $(\tilde{\cdot})$ are omitted in what follows. $\mathbf{F}, \mathbf{G}$ and $\mathbf{H}$ specify the inviscid fluxes in $x$-, $y$ - and $z$-direction, respectively. $\mathbf{F}_{v}, \mathbf{G}_{v}$ and $\mathbf{H}_{v}$ indicates the corresponding viscous fluxes. The source vector

$$
\mathbf{S}=\left[0,0,0,0,0, S_{k}, S_{\omega}, S_{\sigma_{T}}, S_{\sigma_{Y}}, S_{Y_{i}}\right]^{\top}, i=1, \ldots, N_{k}-1
$$

includes terms resulting from turbulence and chemistry modeling. In order to close this set of equations, the ideal gas law is used.

\section{B. Numerical Solver}

A finite-volume approach that works on block-structured meshes is utilized to discretize the Navier-Stokes equations (11). The resulting set of equations is solved with an implicit lower-upper symmetric Gauss-Seidel algorithm [13, 14]. All time-accurate simulations use a second order BDF (backward differentiation formula) scheme [15] with a number of inner Newton iterations.

The inviscid fluxes are calculated with the AUSM+-up flux vector splitting method of Liou [16] originating from the Advection Upstream Splitting Method family [17]. This or any other flux-vector splitting approach requires values for the variables at both sides of the cell interfaces. As described in Sec. [II.C, these values are determined with a fifth/sixth-order scheme. The calculation of the viscous fluxes is done with a central discretization in a cell-oriented coordinate system. Two different hybrid RANS/LES methods (iDDES and $l^{2}-\omega$-DDES) will be compared throughout the course of this paper. 
The simulation of combustion employs finite-rate chemistry. In this work, the hydrogen oxidation kinetic reaction mechanism of Ó Conaire [18] is utilized. This mechanism consists of eight species and 19 reactions. In addition, an assumed probability density function (APDF) approach [19] is used to account for turbulence-chemistry-interaction. Using the assumption of statistical independence of temperature and species fluctuations, the joint pdf of temperature and species composition are simplified into a product of the individual pdfs of temperature and composition. For the first one, a clipped Gaussian distribution which is defined by $T$ and the temperature variance $\sigma_{T}$ is assumed. The joint pdf of all species concentrations is described by a multi-variate $\beta$-distribution using the mean mass fractions and the sum of all species mass fraction variances $\sigma_{Y}$. As can be seen in Eq. 11), additional transport equations for $\sigma_{T}$ and $\sigma_{Y}$ are solved.

\section{Cell Interface Value Reconstruction}

In order to calculate the inviscid fluxes, the values of the primitive variables at the cell interfaces are required. As LES requires a scheme that is not overly dissipative, high-order methods are a promising approach for calculating these values. A high-order scheme can be obtained by polynomial reconstruction. On the other hand, some numerical dissipation may be required which, in the present case, is added by a controlled contribution of total variation diminishing (TVD) type discretization. In order to satisfy the TVD constraint, a multidimensional limiting process with low diffusion $\left(\mathrm{MLP}^{\mathrm{ld}}\right)$ [11, 20] is applied. This technique is an extension of the classical second-order limiters to higher-order schemes. Here, only the basic approach is outlined. Exact details and algorithms can be found in [11, 20, 21].

At the interface $(i+1 / 2, j, k)$ the left, $q_{i+1 / 2, j, k}^{\mathrm{L}}$, and right, $q_{i+1 / 2, j, k}^{\mathrm{R}}$ interface values are required. Here, $q$ is any of the variables in the vector $\mathbf{Q}$ in Eq. (2), the total enthalpy $H$ or the integral specific heat ratio $\bar{\gamma}$. The interface values are reconstructed by

$$
\begin{aligned}
q_{i+1 / 2, j, k}^{\mathrm{L}, \text { unlim }} & =q_{i, j, k}+0.5 \beta_{i, j, k}^{\mathrm{L}} \Delta q_{i-1 / 2, j, k} \\
q_{i+1 / 2, j, k}^{\mathrm{R}, \text { unlim }} & =q_{i+1, j, k}-0.5 \beta_{i+1, j, k}^{\mathrm{R}} \Delta q_{i+3 / 2, j, k}
\end{aligned}
$$

with $\Delta q_{i-1 / 2, j, k}=q_{i, j, k}-q_{i-1, j, k}$. The functions $\beta_{i, j, k}^{\mathrm{L}}$ and $\beta_{i+1, j, k}^{\mathrm{R}}$ determine the order of the scheme. Hence, they include information from various neighbor cells. The discretization stencil of the fifth-order upwind scheme of this work contains three upwind and two downwind cells. In general, the coefficients of these two functions are grid size-dependent and can be derived by polynomial reconstruction. However, they only depend on the grid size. Thus, they do not have to be recalculated in every time step, but can be calculated in advance. In case of an equidistant grid, they are given as

$$
\begin{aligned}
\beta_{i, j, k}^{\mathrm{L}} & =\left(-2 / r_{i-1, j, k}^{\mathrm{L}}+11+24 r_{i, j, k}^{\mathrm{L}}-3 r_{i, j, k}^{\mathrm{L}} r_{i+1, j, k}^{\mathrm{L}}\right) / 30 \\
\beta_{i+1, j, k}^{\mathrm{R}} & =\left(-3 r_{i, j, k}^{\mathrm{R}} r_{i+1, j, k}^{\mathrm{R}}+24 r_{i+1, j, k}^{\mathrm{R}}+11-2 / r_{i+2, j, k}^{\mathrm{R}}\right) / 30 .
\end{aligned}
$$

Here, $r_{i, j, k}^{\mathrm{L}}=\Delta q_{i+1 / 2, j, k} / \Delta q_{i-1 / 2, j, k}$ and $r_{i, j, k}^{\mathrm{R}}=1 / r_{i, j, k}^{\mathrm{L}}$ denote the required slope ratios.

Application of the unlimited interface values of Eq. (4) may lead to oscillations and the occurrence of new maxima and minima. Thus, in order to satisfy the TVD criterion a limiter

$$
\phi\left(r_{i, j, k}^{\mathrm{L}}\right)=\max \left[0, \min \left(\alpha_{x}, \alpha_{x} r_{i, j, k}^{\mathrm{L}}, \beta_{i, j, k}^{\mathrm{L}}\right)\right]
$$

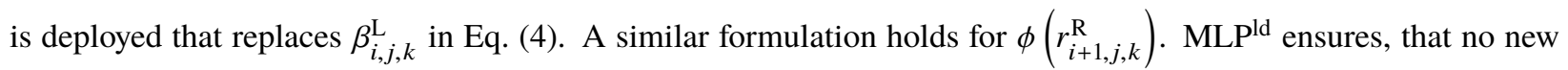
extreme values arise. Hence, the calculation of the parameter $\alpha_{i}(i \in\{x, y, z\})$ in each coordinate direction is the basis for the MLP ${ }^{\mathrm{ld}}$ concept. For that purpose, all surrounding neighbor cells, including those located diagonally, are checked [11,21].

Due to the TVD condition, the MLPld approach may lead to an increased dissipation in instationary flows[22]. With the aid of Eq. (4), an unlimited sixth-order scheme can be defined by averaging of the corresponding left and right unlimited interface values of fifth order. This scheme has almost no dissipation. However, methods that use central discretization stencils tend to be unstable. Therefore, a sensor $S$ is used to define a scheme that blends between an unlimited sixth order central discretization and a limited fifth order upwind discretization.

$$
q_{i+1 / 2, j, k}^{L / R}=q_{i+1 / 2, j, k}^{(6 . O)}+S\left(q_{i+1 / 2, j, k}^{L / R,(5 . O), l i m}-q_{i+1 / 2, j, k}^{(6 . O)}\right),
$$

where $q_{i+1 / 2, j, k}^{(6 . O)}$ is defined as the unlimited sixth-order value. The fifth order upwind biased scheme stabilizes the scheme. In case of non-reactive, subsonic simulations, the sensor $S$ is set to a user-defined value $S=S_{\min }$ that controls the least 
share of limitation. Kindler [23] found that $S_{\min }=0.1$ seems to be advantageous for decaying isotropic homogeneous turbulence. Complex cases often require higher values of $S_{\min }$ in order to be stable.

Besides, due to the highly varying densities of the propellants introduced by a coaxial injector, strong density gradients occur in rocket combustion chambers. These may cause the numerical method to yield spurious oscillations, especially when a high-order, central scheme is applied. One method to suppress the numerical oscillations is to reduce the share of the sixth-order scheme in the cell interface value reconstruction. Here, local density changes are used to determine the location where more dissipation is required. The applied procedure is similar to the one of Hickey et al. [24]. In each direction of each cell, a sensor comparing the density value of the center with the corresponding left and right interface values is evaluated

$$
\max \left(\frac{\left|\rho_{i, j, k}-\rho_{i+1 / 2, j, k}^{L}\right|}{\rho_{i, j, k}}, \frac{\left|\rho_{i, j, k}-\rho_{i-1 / 2, j, k}^{R}\right|}{\rho_{i, j, k}}\right) .
$$

If this sensor is below a certain threshold $R S$, then $S=S_{\min }$. However, if this sensor exceeds $R S$, then a pure fifth order limited scheme is applied, i.e. $S=1$. For the PennState combustor, a value of $R S=0.05$ is adopted.

\section{Hybrid RANS/LES Methods}

Both hybrid RANS/LES methods used in this work rely on the $k-\omega$ turbulence model of Wilcox [25, 26]. The $k-\omega$ equations are given by

$$
\begin{aligned}
\frac{D \rho k}{D t} & =2 \mu_{t}|\mathbf{S}|-\beta_{k} \rho k \omega-\frac{2}{3} \rho k \nabla \cdot \mathbf{u}+\nabla \cdot\left[\left(\mu+\sigma_{k} \rho k / \omega\right) \nabla k\right] \\
\frac{D \rho \omega}{D t} & =\alpha\left(2 \rho|\mathbf{S}|-\frac{2}{3} \rho \omega \nabla \cdot \mathbf{u}\right)-\beta_{\omega} \rho \omega^{2}+\nabla \cdot\left[\left(\mu+\sigma_{\omega} \rho k / \omega\right) \nabla k\right] .
\end{aligned}
$$

Here, $\mu$ and $\mu_{t}$ refer to the molecular and the eddy viscosity, $S$ is the strain tensor and $\sigma_{k}, \sigma_{\omega}$ and $\alpha$ are constants. In DDES type formulations of hybrid RANS/LES methods, these equations are modified in order to enable the formation of eddies. As both applied methods use a different concept in altering these equations, a short description of the differences of these two models is presented in the following subsections.

\section{A. iDDES}

As mentioned above, iDDES, developed by Shur et al. [6], is a further development of DDES. Hence, the general principles are the same. The additional aims are to reduce LLM and to allow the model to act as wall-modeled LES (WMLES) in case of a fine grid and enough turbulence transported from upstream. Identical to DDES, a lower limit is set on the dissipation of the turbulent kinetic energy yielding the following equation

$$
\frac{D \rho k}{D t}=2 \mu_{t}|\mathbf{S}|-\frac{\rho k^{3 / 2}}{l_{D D E S}}-\frac{2}{3} \rho k \nabla \cdot \mathbf{u}+\nabla \cdot\left[\left(\mu+\sigma_{k} \rho k / \omega\right) \nabla k\right] .
$$

The required lengthscale $l_{\text {DDES }}$ is based on the individual lengthscales of the RANS and the LES mode

$$
\begin{aligned}
l_{\text {DDES }} & =f_{\mathrm{d}}\left(1+f_{\mathrm{e}}\right) l_{\mathrm{RANS}}+\left(1-f_{\mathrm{d}}\right) l_{\mathrm{LES}} \\
l_{\mathrm{RANS}} & =\frac{\sqrt{k}}{\beta_{k} \omega} \\
l_{\mathrm{LES}} & =C_{\mathrm{DES}} \Delta \text { with } \Delta=\min \left[\max \left(0.15 d_{\mathrm{w}}, 0.15 h_{\max }, h_{\mathrm{wn}}\right), h_{\max }\right],
\end{aligned}
$$

where $d_{\mathrm{w}}$ is the distance to the wall, $h_{\mathrm{wn}}$ denotes the grid step in wall-normal direction and $h_{\max }$ is defined as the maximum local grid spacing. The definition of the shielding function $f_{\mathrm{d}}$ and the elevating-function $f_{\mathrm{e}}$ can be found in [6]. The turbulent viscosity is calculated as

$$
\mu_{t}=\frac{\rho k}{\omega}
$$




\section{B. $l^{2}-\omega$-DDES}

Recently, another DDES formulation has been published by Reddy et al. [7] and termed $l^{2}$ - $\omega$-DDES. This approach is also based on the $k$ - $\omega$ model, but instead of altering the dissipation term, the production term is modified. Thus, the following equation for $k$

$$
\frac{D \rho k}{D t}=2 \rho l_{D D E S}^{2} \omega|\mathbf{S}|-\beta_{k} \rho k \omega-\frac{2}{3} \rho k \nabla \cdot \mathbf{u}+\nabla \cdot\left[\left(\mu+\sigma_{k} \rho k / \omega\right) \nabla k\right]
$$

is obtained. The values of the constants are chosen according to Wilcox [25]. The lengthscales are defined as

$$
\begin{aligned}
l_{\mathrm{DDES}} & =l_{\mathrm{RANS}}-f_{\mathrm{dt}} \max \left(0, l_{\mathrm{RANS}}-l_{\mathrm{LES}}\right) \\
l_{\mathrm{RANS}} & =\frac{\sqrt{k}}{\omega} \\
l_{\mathrm{LES}} & =C_{\mathrm{DES}} \Delta \text { with } \Delta=f_{\mathrm{dt}} V^{1 / 3}+\left(1-f_{\mathrm{dt}}\right) h_{\max } .
\end{aligned}
$$

Note that both the RANS and the LES lengthscale are different compared to the iDDES formulation. The shielding function $f_{\mathrm{dt}}$ is calculated from

$$
f_{\mathrm{dt}}=1-\tanh \left[\left(8 r_{\mathrm{d}}\right)^{3}\right] \quad \text { with } \quad r_{\mathrm{d}}=\frac{k / \omega+v}{\kappa^{2} d_{\mathrm{w}}^{2} \sqrt{u_{i, j} u_{i, j}}} .
$$

Due to stability issues, a spatial uniformization of the velocity gradient analog to He et al. [27] is applied. In addition, a Smagorinsky-type formulation for $\mu_{t}$ is adopted

$$
\mu_{t}=\rho l_{D D E S}^{2} \omega
$$

Theoretically, this approach allows a dynamic procedure with local computation of the model constant $C_{\mathrm{DES}}$, as described in Yin et al. [8]. Nevertheless, the method used in this work applies a constant value of $C_{\mathrm{DES}}=0.12$.

The main difference between iDDES and $l^{2}-\omega$-DDES is the fact that the former is dissipation-limited, whereas the latter is production-limited. Nevertheless, both procedures pursue the same objectives in enhancing turbulence.

\section{Non-reactive Test Cases}

Before considering a rocket combustion chamber, three non-reactive test cases are simulated in order to validate these two hybrid RANS/LES variants and to identify potential differences. First, decaying isotropic homogeneous turbulence (DIHT) is examined. The other two test cases represent wall-bounded flows, namely a turbulent channel at $R e_{\tau}=5200$ and a backward-facing step flow.

\section{A. Decaying Isotropic Homogeneous Turbulence}

A basic test case to analyze the resolution of turbulent scales of a given numerical method is the DIHT. The conditions correspond to the experiment conducted by Comte-Bellot and Corrsin [28]. DDES simulations are carried out with isotropic grids consisting of $64^{3}$ and $128^{3}$ volumes respectively. The initial velocity field originates from a DNS of Mansour and Wray [29].

An investigation of Kindler [23] showed that the parameter $S_{\min }$ used to switch between a fifth and sixth order scheme should be set to $S_{\min }=0.1$ for this test case. Increasing $S_{\min }$ leads to an overly dissipative method, whereas a smaller value results in too little dissipation and, hence, possible instability of the numerical scheme. Accordingly, the given value of $S_{\min }$ is adopted.

For the DIHT, both iDDES and $l^{2}$ - $\omega$-DDES operate exclusively in LES mode. Since these two methods differ in the LES mode due to Eqs. (15) and (21), the results are different too. Figure 1] depicts energy spectra at a non-dimensional time of $t^{*}=42$ as defined in [28]. This corresponds to a Taylor microscale Reynolds number of $\operatorname{Re}_{\lambda}=71.5$. For small wavenumbers, both DDES models nearly coincide independent of mesh size. However, for wavenumbers close to the maximum, particularly $l^{2}$ - $\omega$-DDES tends to a slight increase in energy. Nevertheless, both iDDES and $l^{2}-\omega$-DDES show a good agreement with experimental and DNS data over a wide range of wavenumbers. 

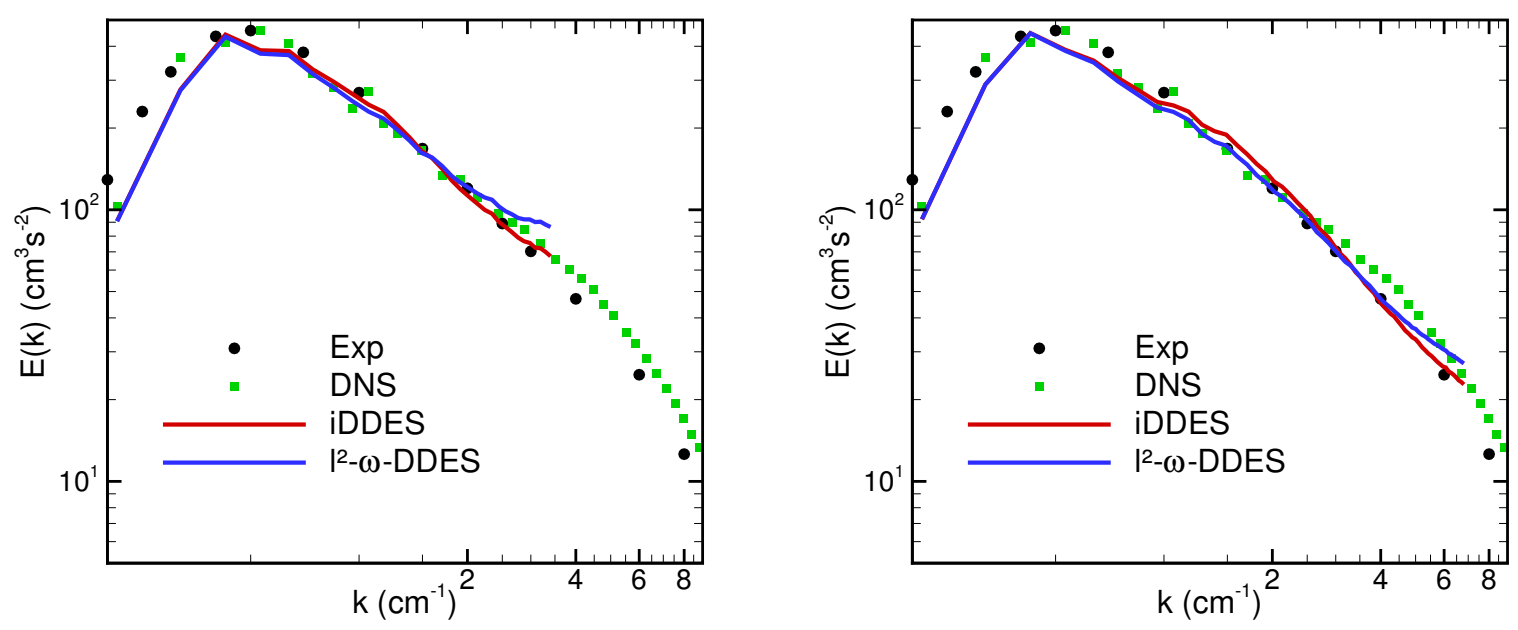

Fig. 1 Comparison of DIHT energy spectra at a non-dimensional time of $t^{*}=42$ predicted by iDDES and $l^{2}$ - $\omega$-DDES. Left: Grid with $64^{3}$ cells; right: Grid with $128^{3}$ cells. Experimental data from Comte-Bellot and Corrsin [28], DNS data from Mansour and Wray [29].

\section{B. Turbulent Channel Flow}

A turbulent channel flow between two flat plates at $R e_{\tau}=5200$ is examined next. The results are compared with DNS data from Lee and Moser [30]. In stream- and spanwise direction, periodic boundary conditions are applied. The channel half width $\delta$ is set to $0.015 \mathrm{~m}$. The grid used for the simulations has $(\Delta x /(2 \delta), \Delta y /(2 \delta))=(0.1,0.05)$. This corresponds to a grid resolution of $\left(\Delta x^{+}, \Delta z^{+}\right)=(1040,520)$ in wall units. The volumes next to the walls satisfy $\Delta y^{+}<1$. The total amount of volumes is about 461000 . For pure LES purposes, this grid would be rather coarse. However, the grid spacing is typical for hybrid RANS/LES simulations [6]. Since the objective of these calculations is not necessarily to obtain the best possible result, but to compare the two DDES methods, this grid is considered to be sufficient.

Non-dimensional mean velocity profiles are depicted in Fig. 2 a). The velocity is normalized with the friction velocity $u_{\tau}$. Near the walls, both models nearly coincide. However, in the log-layer, iDDES slightly underpredicts the DNS data, whereas the $l^{2}$ - $\omega$-DDES matches these perfectly. Near the middle of the channel both models overestimate the velocity.

Figure 2 b) shows the ratio of turbulent to laminar viscosity along the wall-normal direction. In addition, this figure includes the DES mode. This quantity indicates whether the method is in RANS (DES mode $=0$ ) or in LES mode (DES mode $=1$ ). Anything in between corresponds to the gray area. In case of the channel flow, the DES mode agrees with the corresponding shielding functions of the iDDES and the $l^{2}-\omega$-DDES approach. This does not have to apply in general, because a too large LES length scale $l_{\text {LES }}$ may force the model to operate in RANS mode even though the shielding function would enable LES. Both methods use the $k$ - $\omega$-model as underlying RANS model. Hence, the turbulent viscosity of iDDES and $l^{2}-\omega$-DDES coincides for the RANS mode. However, as soon as the models deviate from pure RANS, the turbulent viscosity diminishes. As this occurs closer to the wall for the iDDES, the maximal tubulent viscosity of the iDDES is significantly smaller than the one of the $l^{2}-\omega$-DDES. Since the gray area of the $l^{2}-\omega$-DDES is smaller, the turbulent viscosity decreases faster for that method. Near the center of the channel, where LES prevails, the turbulent viscosity of $l^{2}$ - $\omega$-DDES adopts clearly higher values compared with iDDES. The values differ by approximately one order of magnitude. This is caused by the different definitions of the turbulent viscosity, see Eq. (15) and Eq. 21.

Figure $2 \mathrm{c}$ ) displays the total normalized shear stresses as well as the corresponding modeled (subgrid-scale) and resolved parts. Both models accurately predict the total shear stress. However, over the entire channel height, the absolute value of the resolved part of the $l^{2}-\omega$-DDES is lower than that of the iDDES. In contrast, the contribution of the modeled part to the total shear stress is greater for the former. This behavior does not necessarily mean that iDDES outperforms $l^{2}-\omega$-DDES. Instead, this is a consequence of the reduced turbulent viscosity of iDDES and the 
a)

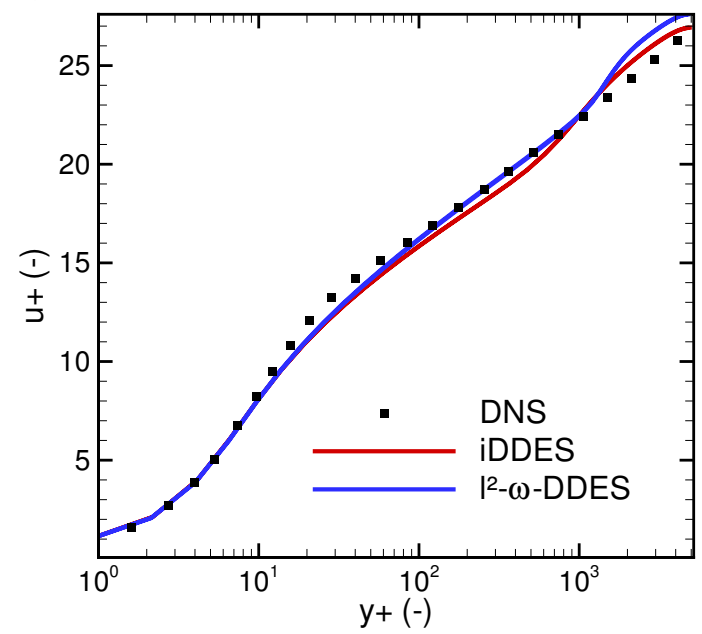

c)

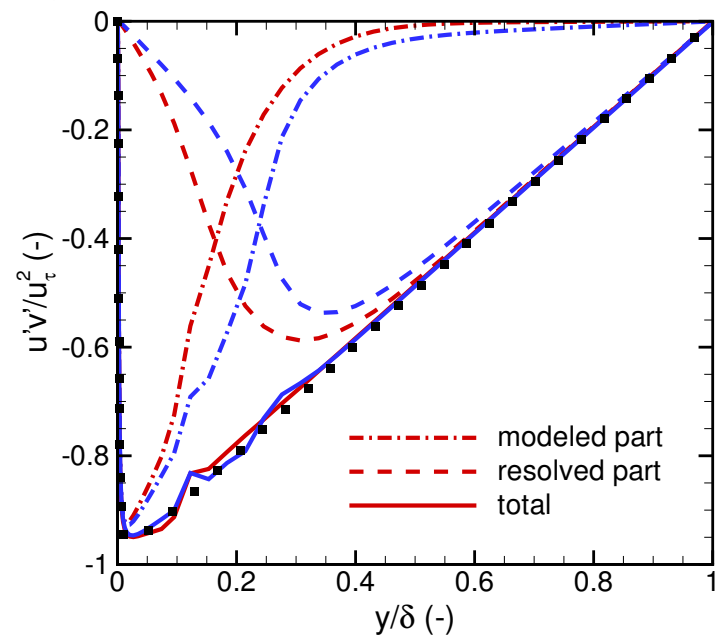

b)

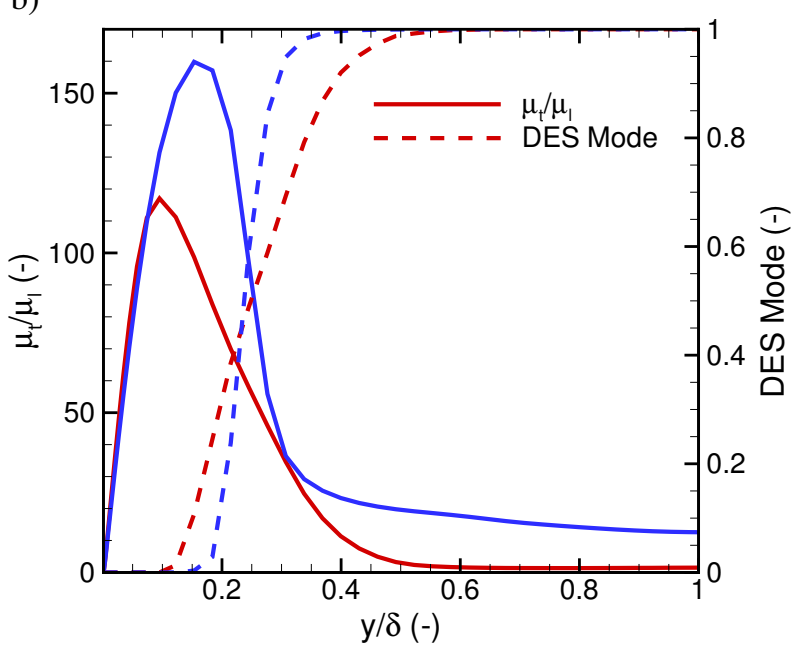

d)

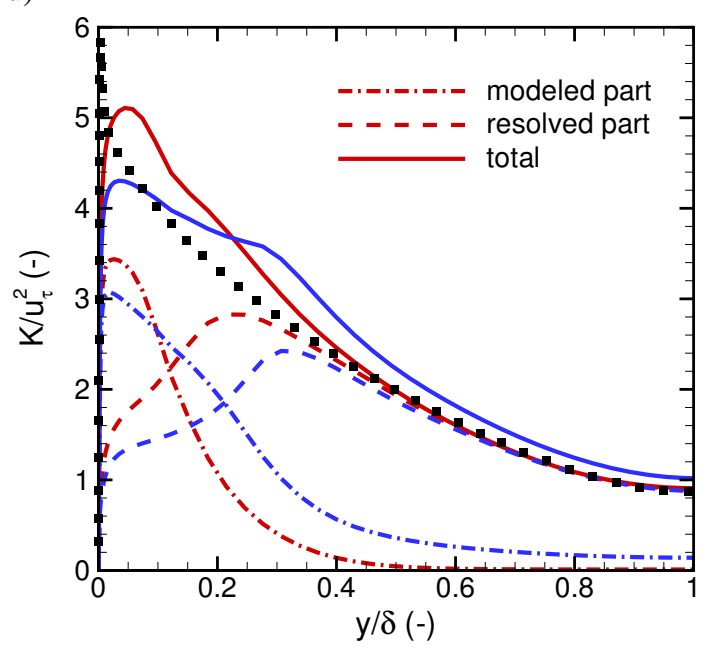

Fig. 2 a) Non-dimensional mean velocity profiles; b) Ratio of turbulent to laminar viscosity and DES mode; c) Non-dimensional shear stress; d) Turbulent kinetic energy. DNS data from Lee and Moser [30].

correspondingly reduced subgrid-scale shear stress. Time-averaging of the streamwise momentum equation yields the relationship $y / \delta-1$ for the total shear stress [31]. Hence, the resolved shear stress balances the modeled one, thus leading to higher magnitude of the resolved shear stress for the iDDES.

The total turbulent kinetic energy is depicted in Fig.2 d). It is also composed of a resolved and a modeled part and is defined as

$$
K=\frac{1}{2}\left\langle u_{i}^{\prime} u_{i}^{\prime}\right\rangle+\langle k\rangle
$$

where $u_{i}^{\prime}$ indicates the fluctuating part of the velocity component $i$. The turbulent kinetic energy $k$ calculated by the Eqs. (11) and (16) represents the modeled part. Time-averaging is denoted by $\langle\cdot\rangle$. The peak next to the wall in the DNS data is not reproduced correctly by any of the models. This peak occurs in an area where both models are in pure RANS mode. Accordingly, the resolved part is too small to capture this effect. In addition there are the shortcomings arising from Boussinesq's hypothesis. Similar to the shear stress in Fig. 2]c), the magnitude of the resolved part of the iDDES is higher than the one of the $l^{2}-\omega$-DDES. However, near the channel half width they approach each other. For $y / \delta \geq 0.1$ the modeled part of the turbulent kinetic energy of the $l^{2}-\omega$-DDES exceeds the one of the iDDES and therefore has a significant share to the total turbulent kinetic energy over the entire channel height. This behavior is due to the different formulations of the dissipation-limited iDDES and the production-limited $l^{2}-\omega$-DDES. Although the resolved part of 

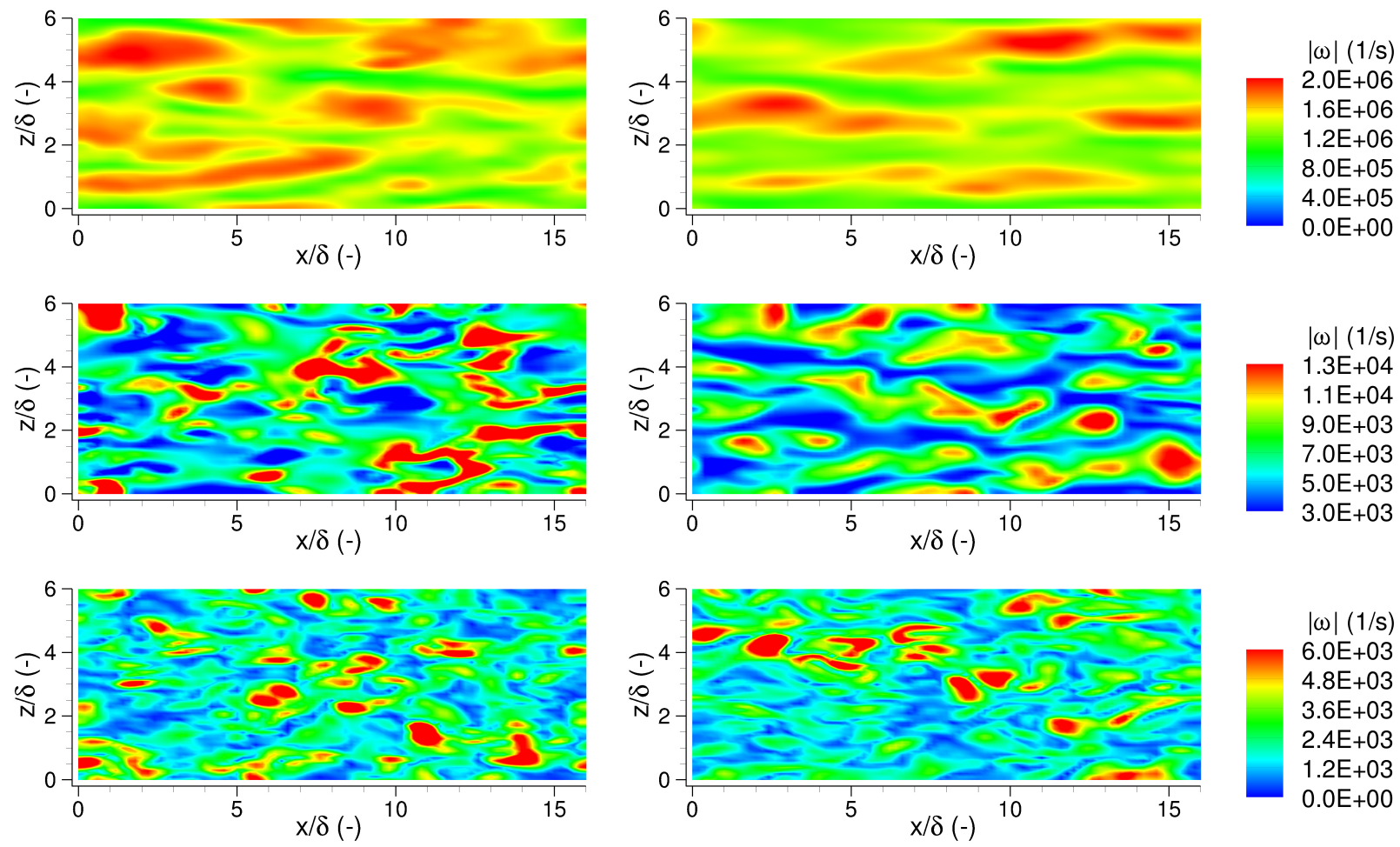

Fig. 3 Comparison of instantaneous vorticity magnitude. Left: iDDES; Right: $l^{2}-\omega$-DDES. From top to bottom $y / \delta=5 \times 10^{-4}, y / \delta=0.23, y / \delta=1.0$.

the $l^{2}$ - $\omega$-DDES coincides with the DNS data in the range $0.6 \geq y / \delta \geq 1.0$, the modeled part leads to an overestimation of $K$. Here, the iDDES behaves better as the total kinetic energy matches the DNS data for $y / \delta \geq 0.4$.

Figure 3 shows the vorticity magnitude at three different planes parallel to the wall. On the left-hand side the results from the iDDES are depicted. The right-hand side illustrates the $l^{2}$ - $\omega$-DDES. The top row corresponds to a plane with a normalized distance to the wall of $5 \times 10^{-4}$ and is clearly located in the RANS regime. The occurring streaks are rather elongated, nearly forming so-called "super-streaks" [32]. Nevertheless, the structures of the iDDES seem to be slightly more fine-scaled. In contrast to this, the two models behave differently at $y / \delta=0.23$, which is located at the RANS/LES interface. The eddies forming in the iDDES are not smooth and one-dimensional, whereas the $l^{2}$ - $\omega$-DDES still predicts elongated streaks. However, these are not as pronounced as the ones at the plane next to wall. At the center of the channel, shown at the bottom of Fig. 3 . both models show similar properties.

A more quantitative assessment of the above observations is obtained by streamwise two-point correlations, which are depicted in Fig. 4] The two-point correlation, as defined by Pope [33], is calculated from

$$
B_{u u}(x)=\left\langle u^{\prime}\left(x_{0}\right) u^{\prime}\left(x_{0}+x\right)\right\rangle,
$$

and, in this case, is independent of the location $x_{0}$. The two-point correlations are normalized with the respective $u_{\mathrm{rms}}^{2}$ and are an indicator of the streamwise extent of the eddies. In contrast to the observations by Davidson [31], they vary across the channel, especially for the $l^{2}-\omega$-DDES case. Closer to the channel wall, the two-point correlation increases, because smaller scales are not resolved correctly. The two-point correlation of the $l^{2}-\omega$-DDES at $y / \delta=0.23$ clearly shows the unphysical behavior in the gray area as a quarter of the channel width is required to decrease to a value of 0.4. Besides, the two-point correlation of the $l^{2}-\omega$-DDES is higher than the one of the iDDES. This can be attributed to the increased turbulent viscosity.

\section{Backward-facing Step}

The last non-reacting test case is a backward facing step flow experimentally investigated by Driver and Seegmiller [34] and Driver et al. [35]. This is a rather important test case as the hybrid RANS/LES models must switch from RANS to 


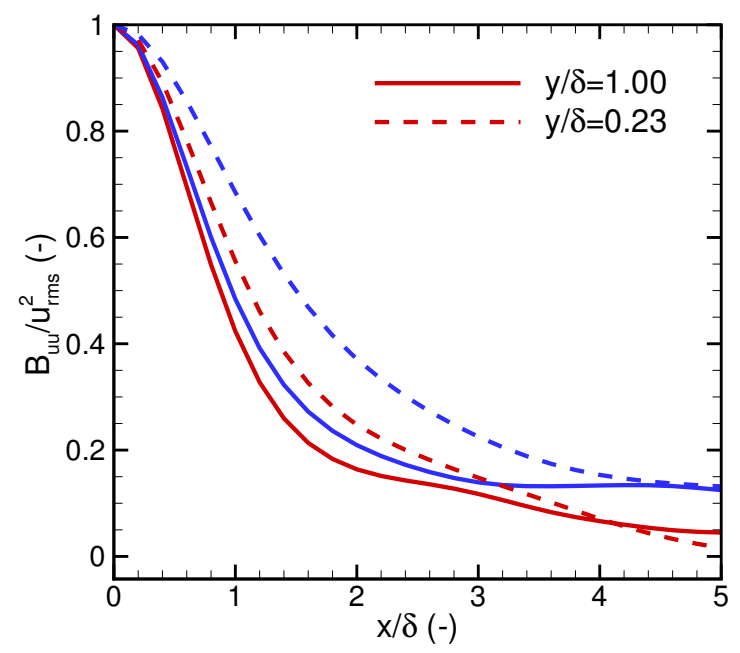

Fig. 4 Normalized two-point correlations of iDDES and $l^{2}$ - $\omega$-DDES for two different distances to the wall. Dashed line: $y / \delta=0.23$; Solid line: $y / \delta=1.0$. For coloring, see legend of Fig. 2$]$ a).
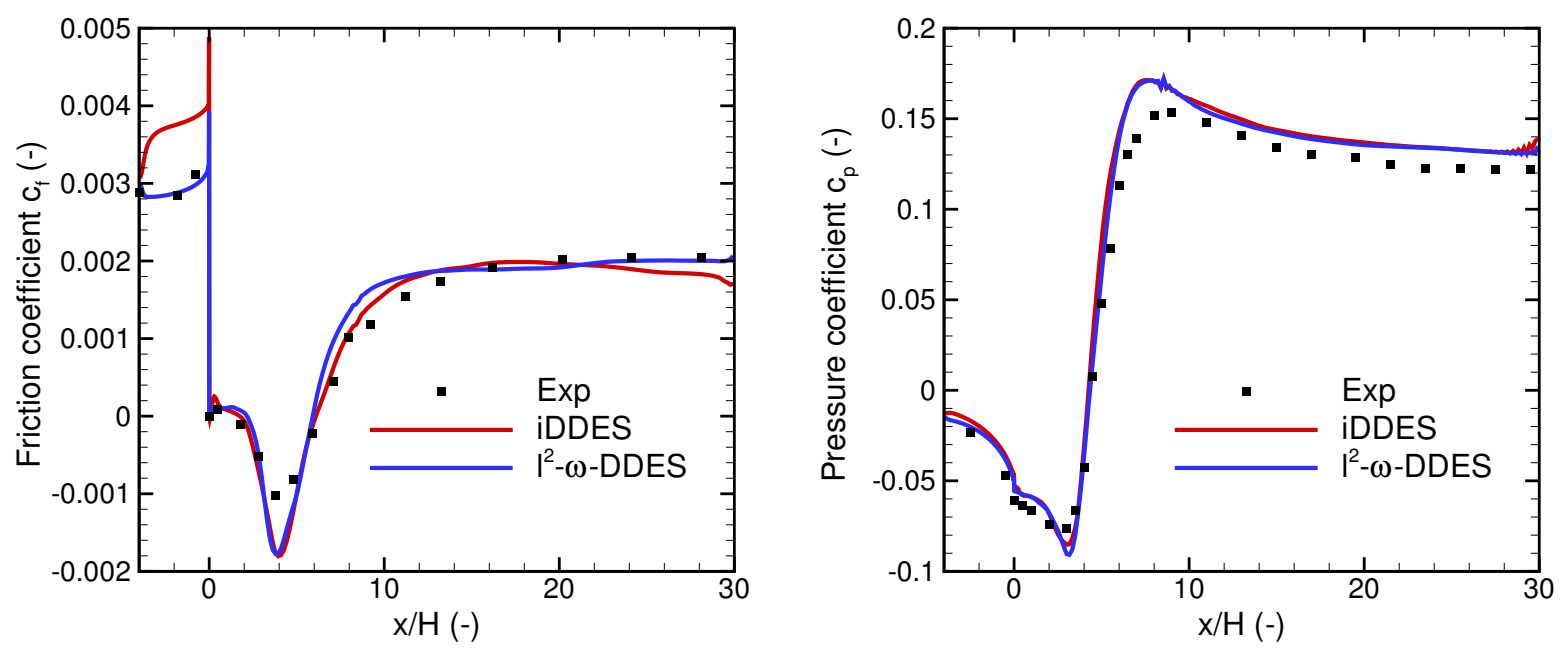

Fig. 5 Left: Skin-friction coefficient $c_{\mathrm{f}}$ at the lower wall; right: Wall static pressure coefficient $c_{\mathrm{p}}$. Experimental data are from Driver and Seegmiller [34].

LES mode at the step, which defines the point of separation, without any initial perturbations. Besides, at the upper channel wall as well as upstream of the step, the models should be in pure RANS mode. The step height is $H=1.27 \mathrm{~cm}$ and it is located at $x / H=0$. Air enters the domain through an inflow duct with height $8 H$ at a reference velocity of $u_{\mathrm{b}}=44.2 \mathrm{~m} / \mathrm{s}$. The simulation domain starts $4 H$ upstream of the step and extends up to $30 H$ downstream. The spanwise extent is $4 H$. Periodic boundary conditions are applied in this direction. The inflow is prescribed by a preceding steady-state RANS simulation that matches the experimental boundary layer thickness.

The mesh consists of a total of 1.38 million volumes. Thirty uniformly spaced cells are used for the spanwise direction, while the step is resolved with 80 volumes. The mesh and the boundary conditions are identical for both DDES variants.

The left-hand side of Fig. 5 depicts the skin friction coefficient $c_{\mathrm{f}}$ at the lower wall. Between the step and the reattachment point, iDDES and $l^{2}-\omega$-DDES show only slight differences. Both models underestimate the minimal 


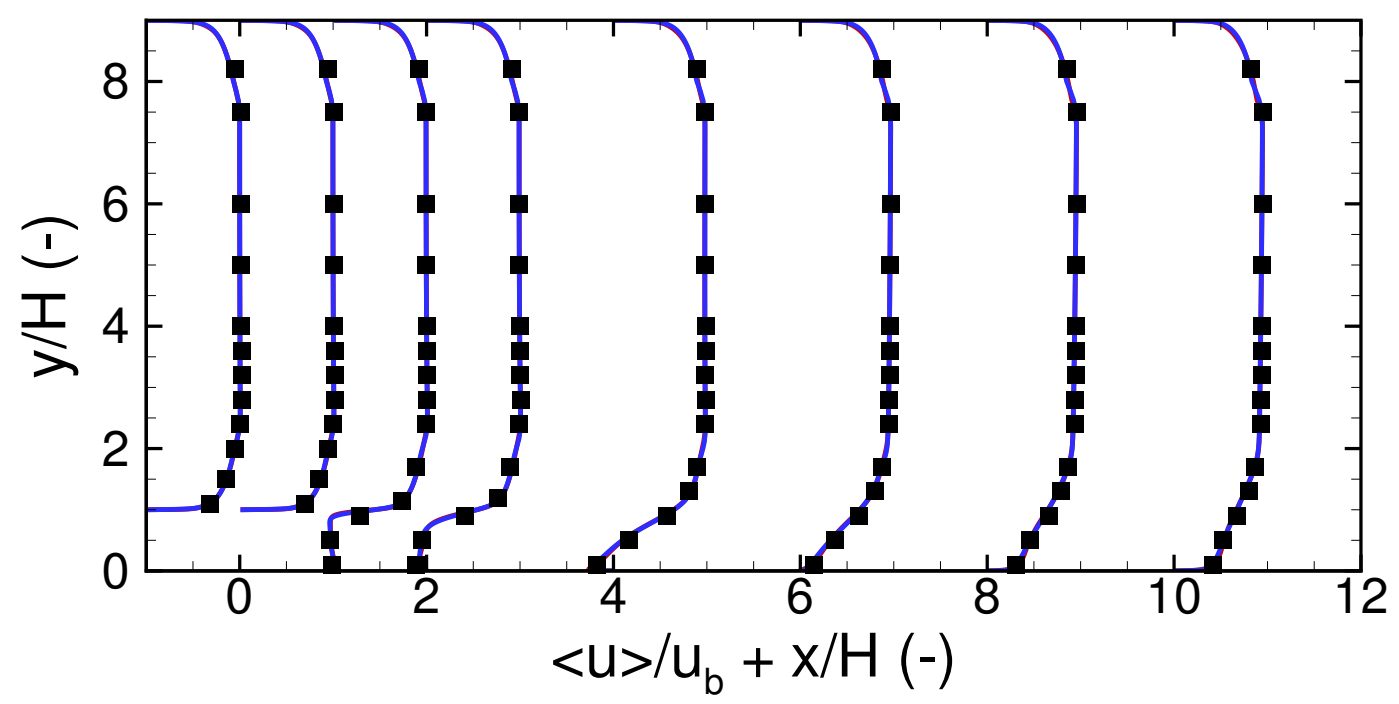

Fig. 6 Mean streamwise velocity normalized by $u_{\mathbf{b}}$. Same legend as Fig. 5 . Experimental data are from Driver and Seegmiller [34]. Profiles are plotted at $x / H=-1.0,0.0,1.0,2.0,4.0,6.0,8.0,10.0$.

experimental skin friction coefficient. Likewise, the predicted reattachment lengths are too small and deviate by $2.6 \%$ (iDDES) and 5.5\% ( $l^{2}-\omega$-DDES) from the experimental value of $6.26 H$. Downstream of the reattachment point, iDDES and $l^{2}-\omega$-DDES deviate. The $l^{2}$ - $\omega$-DDES exhibits a too steep increase in the range between $x / H=6.26$ and $x / H \approx 9.0$, whereas the iDDES underestimates the measured skin friction in the rear part of the numerical domain. The static pressure coefficient at the lower wall is displayed on the right-hand side of Fig. 5 Both DDES variants differ only slightly from each other. However, compared to the experimental data, a deviation is observable downstream of the reattachment point.

Figure 6 shows the mean streamwise velocity at eight different locations. Again, iDDES and $l^{2}-\omega$-DDES agree quite well with each other. Small differences arise near the walls. Despite the deviations in skin friction and pressure coefficients, the agreement of both models with the experimental data is excellent. This also applies to positions with $x / H>10$ (not shown here).

The ratio of turbulent to laminar viscosity is depicted in Fig. 7. In addition, this figure includes the isoline where the corresponding model is $90 \%$ in LES mode. As required, iDDES and $l^{2}-\omega$-DDES operate in RANS mode at the upper wall as well as at the lower wall upstream of the step. The height of the RANS mode is nearly the same for both models. Somewhat downstream of the step, both models switch from RANS to LES. This delay of transition, which is typical for such kind of simulations [36], is more pronounced in case of the iDDES. Accordingly, the eddy viscosity values are higher in that area. However, at the lower wall, the WMLES capabilities of the iDDES take effect, thus leading to an extremely narrow RANS range. In contrast, the $l^{2}$ - $\omega$-DDES shows a somewhat broader RANS range. In addition, the RANS mode of the $l^{2}$ - $\omega$-DDES is shaped irregularly and strongly time-dependent due to the turbulent nature of the flow downstream of the step. The eddy viscosity of this model in this region is still lower than upstream of the step, thereby enabling the formation of vortices. Compared with the iDDES, $l^{2}-\omega$-DDES exhibits higher levels of eddy viscosity downstream of the step. This also has a significant effect on the instantaneous flow field.

Contour plots of the instantaneous vorticity magnitude in the xz-plane at half step height are depicted in Fig. 8 . Similar to the channel flow, the vortex structures of the iDDES are significantly smaller than the ones of the $l^{2}-\omega$-DDES. This is due to the higher level of eddy viscosity. It is important to note that, as shown above, this smaller scale resolution of turbulent structures does not lead to an improved prediction of mean flow quantities. 


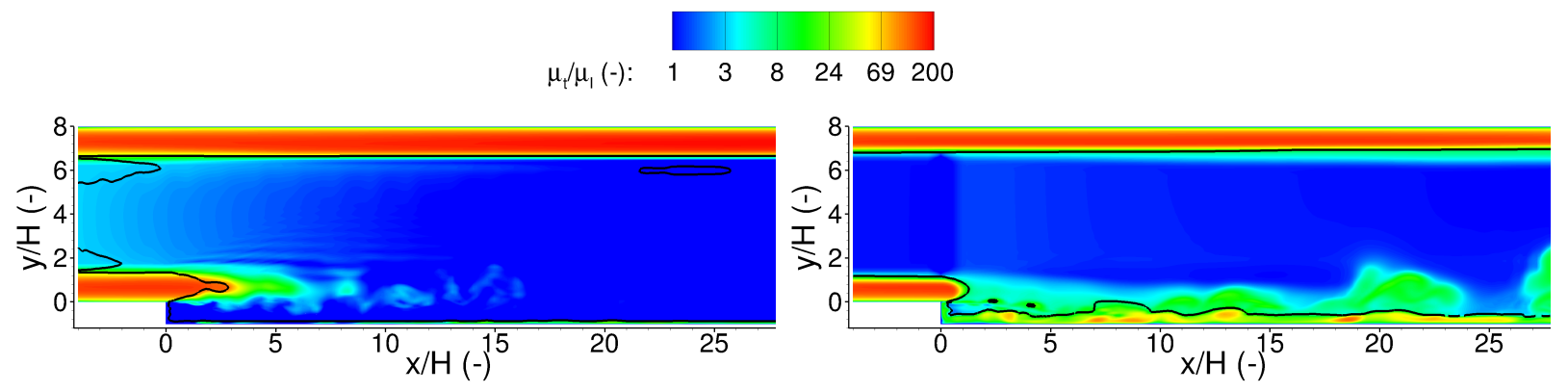

Fig. 7 Contour plot of eddy viscosity. Black line indicates the isoline where the corresponding model is $90 \%$ in LES mode. Left: iDDES; Right: $l^{2}-\omega$-DDES. Note that a non-linear scale for the contour levels is used.

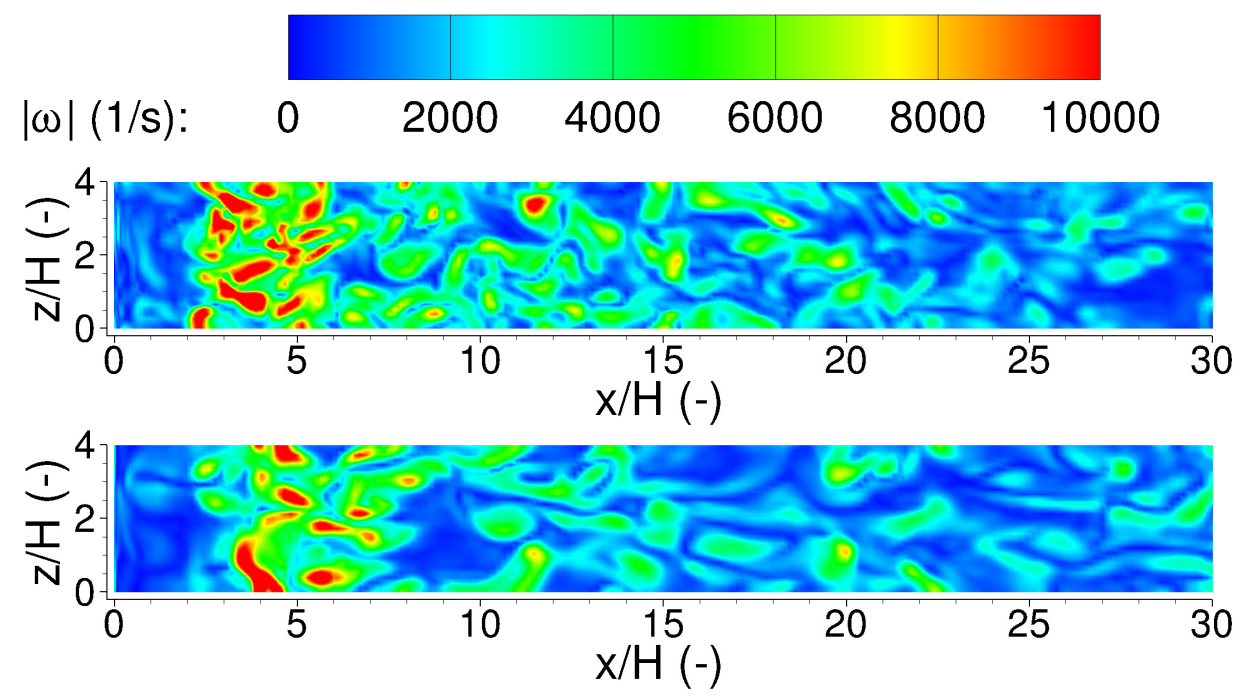

Fig. 8 Instantaneous vorticity magnitude of BFS flow at half step height $(y / H=-0.5)$. Top: iDDES; Bottom: $l^{2}-\omega$-DDES.

\section{PennState Preburner Combustor}

\section{A. Test Case Description}

The PennState preburner combustor is a frequently simulated combustion chamber, which has been investigated both with RANS/URANS and LES by various groups, e.g. [21, 37, 38]. It has been experimentally examined at the Pennsylvania State University with the goal to provide data for the verification and validation of numerical codes [39]. The combustion chamber is axisymmetric and exhibits a single coaxial injector. The chamber length is $285.75 \mathrm{~mm}$, its diameter $38.1 \mathrm{~mm}$ and the diameter of the nozzle throat $8.2 \mathrm{~mm}$. Gaseous oxygen and gaseous hydrogen are preburned in an oxidizer- and fuel-rich preburner respectively and then are supplied to the combustion chamber with a global O/F of $\sim 6.6$. The oxidizer post is recessed $0.43 \mathrm{~mm}$ compared with the faceplate. Gas composition and flow parameters of the oxidizer and fuel stream are listed in Tab.1 A more detailed description can be found in [39].

The computational grid consists of approximately 19.6 million volumes, divided into 39 blocks. The injector including the recess area accounts for $5.4 \%$ of the cells. All other volumes are for the combustion chamber and the nozzle. In radial direction, the oxidizer post tip is resolved by 16 volumes. Apart from exceptions at the faceplate and shortly downstream of it, the wall grid spacing in radial direction of the chamber satisfies $\Delta y^{+}<1$. The experimental data set consists of wall temperature and wall heat fluxes. While the latter serves as validation data, the former is used to prescribe the temperature at the combustion chamber wall. The injector walls as well as the faceplate are assumed to be adiabatic. At the inlet, a mass flow rate is specified. A supersonic outflow condition is imposed at the outlet.

Both simulations are performed with a constant time step of $\Delta t=10^{-8} \mathrm{~s}$. The results are time-averaged over $0.0112 \mathrm{~s}$ 
Table 1 Flow parameter of the oxidizer and fuel streams, respectively.

\begin{tabular}{|c|c|c|}
\hline & Oxidizer Stream & Fuel stream \\
\hline Gas composition & $0.945\left(\mathrm{O}_{2}\right)$ & $0.402\left(\mathrm{H}_{2}\right)$ \\
& $0.05545\left(\mathrm{H}_{2} \mathrm{O}\right)$ & $0.598\left(\mathrm{H}_{2} \mathrm{O}\right)$ \\
Total mass flow rate $(\mathrm{kg} / \mathrm{s})$ & 0.0904 & 0.0331 \\
Temperature $(\mathrm{K})$ & 700 & 811 \\
Bulk velocity $(\mathrm{m} / \mathrm{s})$ & 154 & 764 \\
Reynolds number & 604,000 & 169,000 \\
Inner diameter $(\mathrm{mm})$ & - & 5.26 \\
Outer diameter $(\mathrm{mm})$ & 6.30 & 7.49 \\
\hline
\end{tabular}

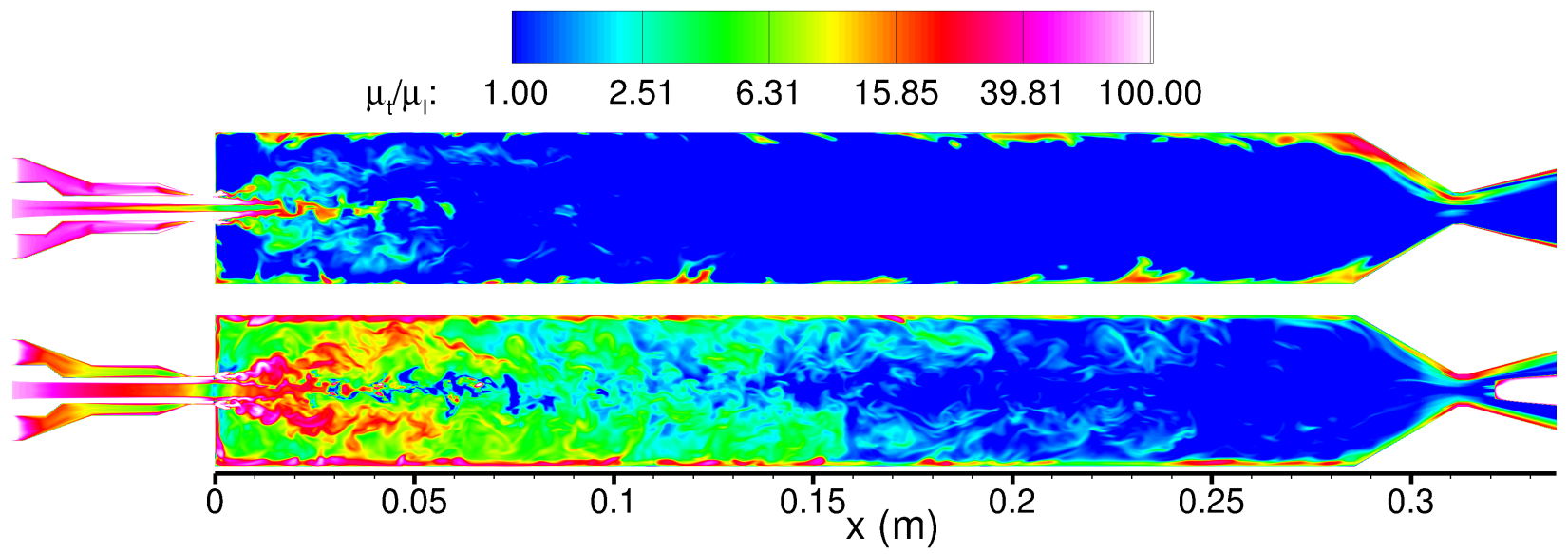

Fig. 9 Ratio of turbulent to molecular viscosity in the center-plane of the PennState preburner combustor using iDDES (top) and $l^{2}$ - $\omega$-DDES (bottom). Note that a non-linear scale for the contour levels is used.

and $0.00307 \mathrm{~s}$ for the iDDES and the $l^{2}-\omega$-DDES case, respectively. Using a flow through time of $8.3 \mathrm{~ms}$ as defined by Tucker et al. [37], this corresponds to 1.35 and 0.37 flow through times. These are rather short averaging periods and especially the $l^{2}-\omega$-DDES is not fully converged. Nevertheless, the general trend in the results is already observable.

\section{B. Results}

At first, Fig. 9 compares the turbulent viscosity of both approches. Similar to the non-reacting test cases, the $l^{2}$ - $\omega$-DDES exhibits higher levels of turbulent viscosity within the whole combustion chamber. As the turbulent viscosity appears in the APDF transport equations, not only the flow field but also the combustion process is influenced. Both models exhibit its highest values of turbulent viscosity shortly downstream of the injector lip. In case of the iDDES, these high values decay quickly. With $l^{2}-\omega$-DDES, however, high values of turbulent viscosity extend up to the chamber wall, thus affecting the recirculation zone.

Figure 10 shows the instantaneous temperature distributions from both methods in the center-plane. Behind the injector lip, for approximately half the diameter of the oxidizer stream, no coherent vortical structures are visible. Masquelet [40] attributes this behavior to the stability of the high speed hydrogen stream. However, as such a behavior is not existent in the pure LES of Oefelein [41], this might be an artifact of the hybrid RANS/LES models where the switching from RANS to LES mode in that region slightly hinders the production of turbulence. Further downstream, typical Kelvin-Helmholtz instabilities occur, causing small-scale vortical structures. The cold oxygen core breaks down at around $x \approx 50 \mathrm{~mm}$. This is similar to the simulations of Ma et al. [38]. Due to the comparably high wall-injector distance that is not present in real-world combustors, a rather large recirculation zone exists at the corner of the combustor. It extends up to approximately one third of the chamber length and is formed from the hydrogen jet and the 


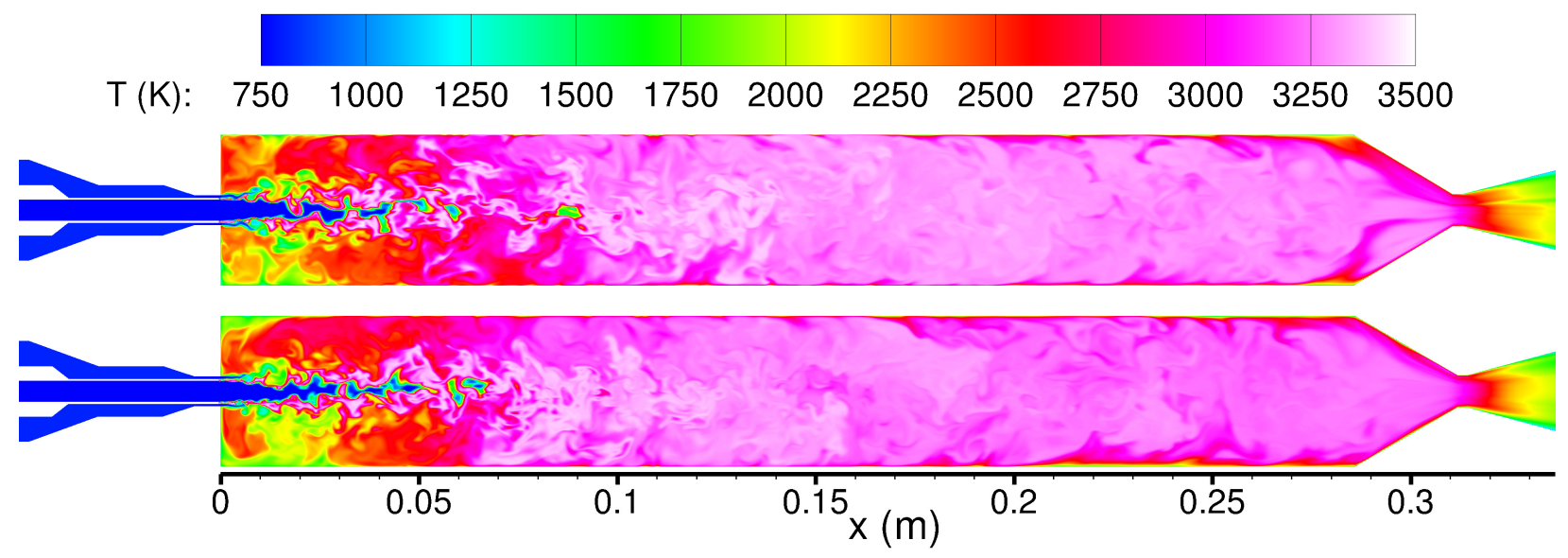

Fig. 10 Instantaneous temperature distribution of the PennState preburner combustor using iDDES (top) and $l^{2}$ - $\omega$-DDES (bottom).

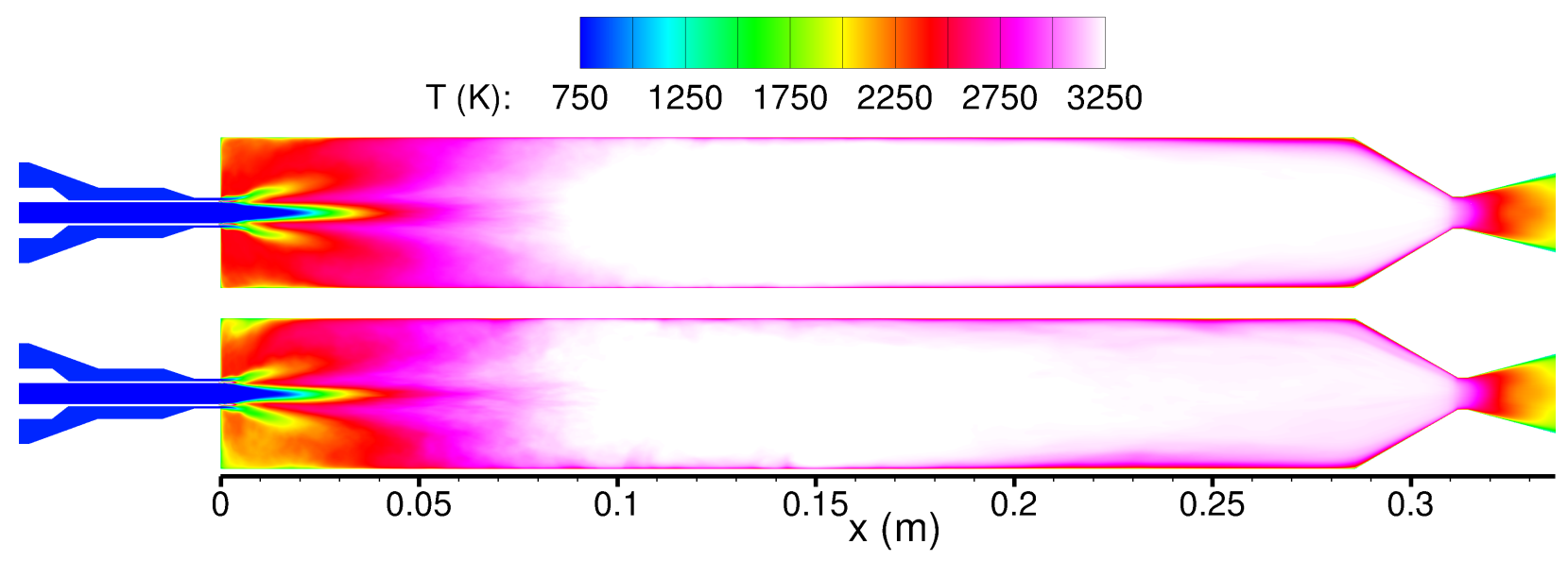

Fig. 11 Time-averaged temperature distribution using iDDES (top) and $l^{2}-\omega$-DDES (bottom).

hot combustion products.

The time-averaged temperature distribution is depicted in Fig. 11. Only slight differences appear between both models. The most remarkable one is the still existing asymmetry in the $l^{2}$ - $\omega$-DDES showing the necessity of further time-averaging. Besides, the temperature of the iDDES in the recirculation zone is slightly higher than the one of the $l^{2}$ - $\omega$-DDES. However, this only holds for the temperature inside the recirculation zone. In the wall-nearest volumes of that zone, the averaged temperature of the $l^{2}-\omega$-DDES reaches higher values. In the rear part, a nearly homogeneous temperature distribution exists.

The mass fraction of hydrogen and oxygen are depicted in Fig. 12 and Fig. 13 , respectively. In both cases, the cold oxygen core shows a similar behavior. Using an arbitrary cutoff of $10 \%$ oxygen mass fraction, the oxygen core has a length of $0.084 \mathrm{~m}$ in the iDDES case and a length of $0.083 \mathrm{~m}$ in the $l^{2}-\omega$-DDES case. In the recirculation zone of the $l^{2}$ - $\omega$-DDES, more hydrogen persists. These higher levels of hydrogen might be a consequence of the increased level of turbulent viscosity in this case. This leads to an increased transport of hydrogen into the recirculation zone. All in all, the differences appear to be small. However, they are noticeable and have an impact on quantities like the wall heat flux. The comparison of the mass fraction of $\mathrm{H}_{2} \mathrm{O}$ does not reveal any significant differences. Therefore, these results are omitted here.

Figure 14 depicts the $\mathrm{OH}$ distribution. Throughout the whole chamber, iDDES shows slightly higher levels of 


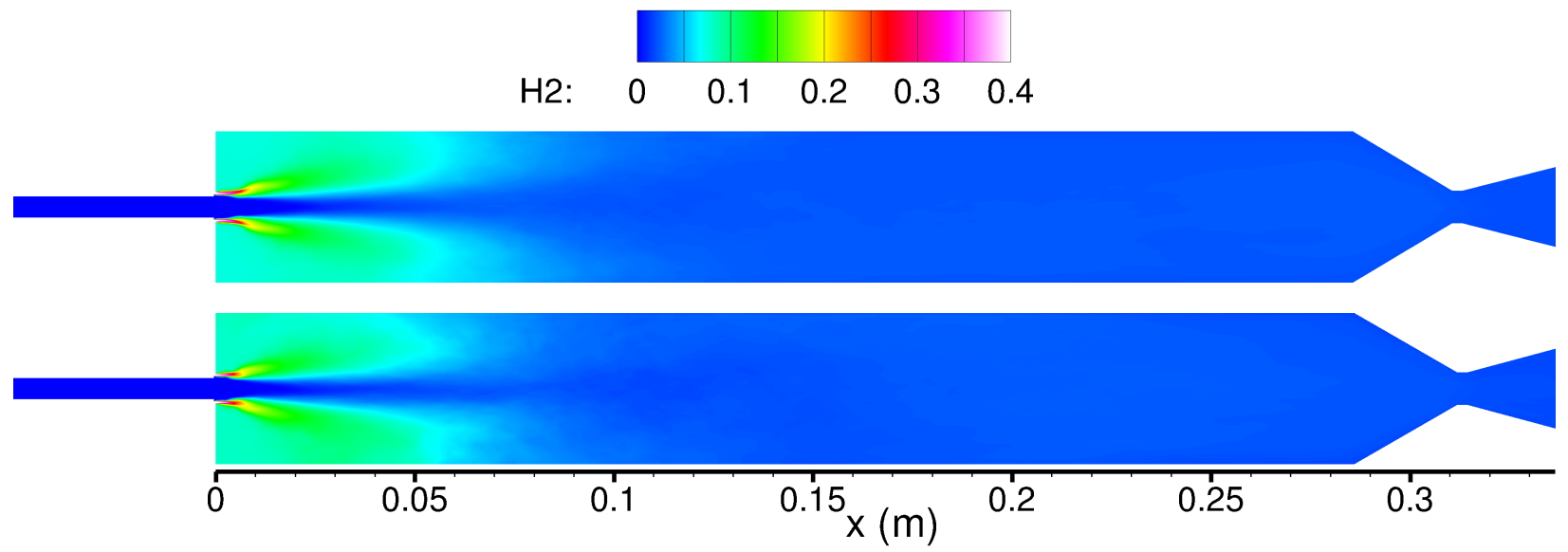

Fig. 12 Comparison of time-averaged hydrogen mass fraction using iDDES (top) and $l^{2}-\omega$-DDES (bottom).

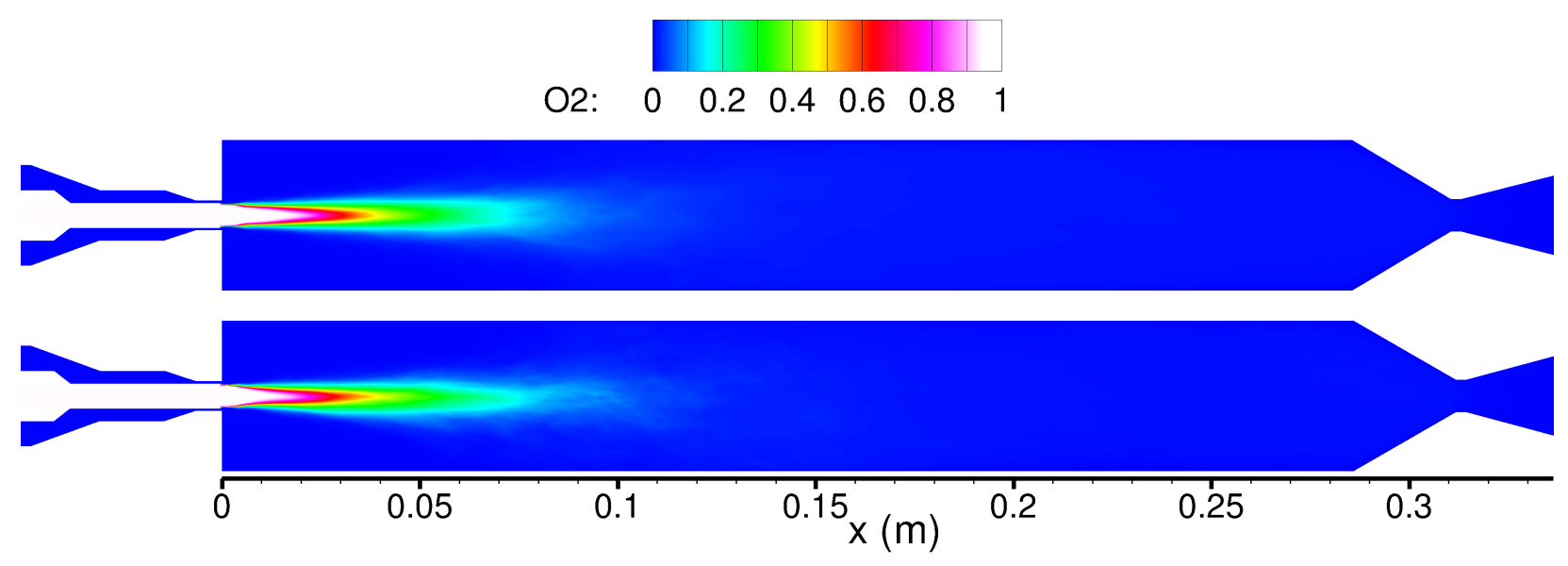

Fig. 13 Mean oxygen mass fraction for iDDES (top) and $l^{2}-\omega$-DDES (bottom).

hydroxyl mass fraction indicating differences in the combustion process. Although the adopted values of hydroxyl mass fraction differ, the general flame zones are similar.

The wall heat fluxes of both simulations are shown in Fig. 15 and compared to the experimental data of Marshall et al. [39]. In addition to time-averaging, these profiles are also averaged in azimuthal direction. The iDDES reaches a maximal wall heat flux of $12.33 \mathrm{MW} / \mathrm{m}^{2}$ which is approximately $25 \%$ below the experimental value. However, the location coincides very well with the measured one. Downstream of the maximal value, the wall heat flux of the iDDES decreases at a nearly constant slope. This is in contrast to the experiment. After the plateau that extends up to the end of the recirculation zone, there is a steeper slope up to the middle of the combustion chamber. Further downstream, the decrease is flatter. The $l^{2}-\omega$-DDES nearly matches the experimental peak. Besides, this approach predicts first a steeper, then a flatter decline of the wall heat flux. However, the plateau existent in the experimental data set is not predicted. In the rear part, both approaches coincide with the experiment. The wall heat flux depends on the radial temperature gradient at the wall as well as the heat conduction coefficient of the mixture. Hence, a correct prediction of the temperature and the gas composition in the wall-nearest volume is required. On average, the simulated wall temperature of the $l^{2}$ - $\omega$-DDES exceeds the one of the iDDES in the recirculation zone by maximal $10 \mathrm{~K}$. Around $x \approx 200 \mathrm{~mm}$, the wall temperature gradient of the iDDES is slighlty higher than the one of the $l^{2}-\omega$-DDES. This explains the slight discrepancies between the two hybrid RANS/LES models in the range of $x \approx 150-230 \mathrm{~mm}$, but not the differences in the front part. These are due to different mass fractions of $\mathrm{H}_{2}$ as well as $\mathrm{H}$ in the recirculation zone, see 


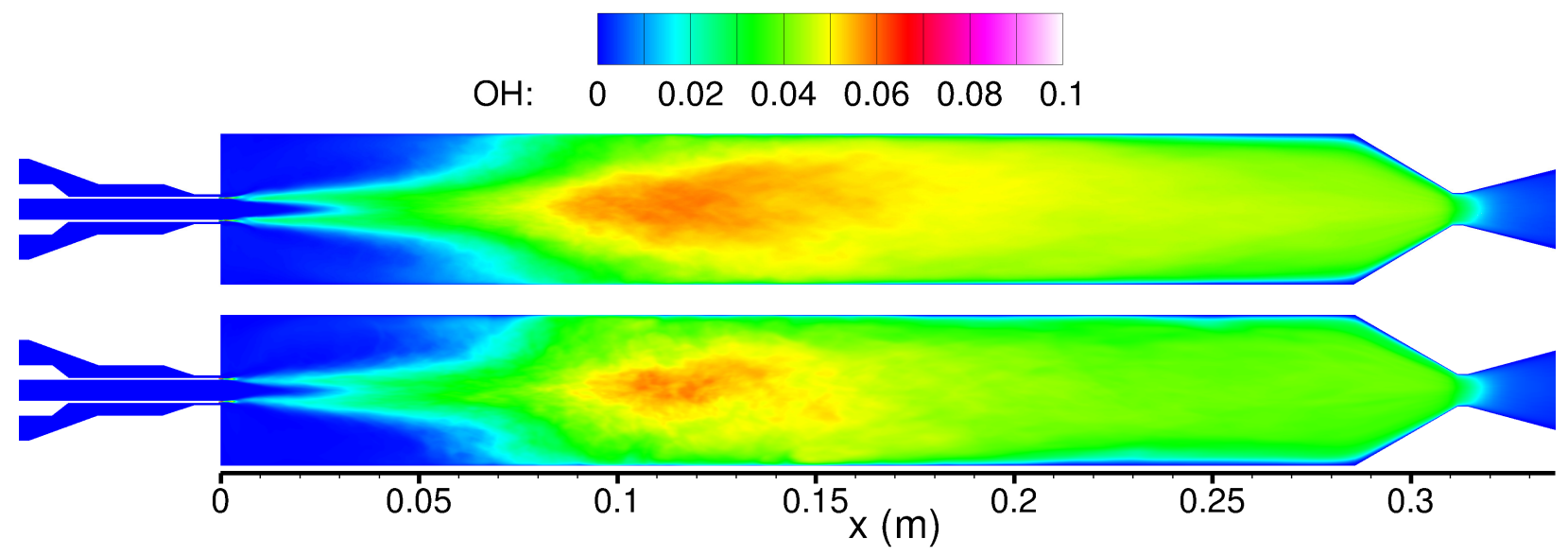

Fig. 14 Instantaneous $\mathrm{OH}$ mass fraction distribution of the PennState preburner combustor using iDDES (top) and $l^{2}-\omega$-DDES (bottom).

Fig. 12 Compared with the experiment, the $l^{2}-\omega$-DDES performs significantly better than the iDDES.

\section{Conclusions}

In this paper, the dissipation-limited iDDES and the production-limited $l^{2}-\omega$-DDES were compared to each other. Four test cases, including one rocket combustion chamber, were simulated and investigated.

For the non-reactive test cases, mean quantities of both models show similar results. In addition, these results agree quite well with experimental or DNS data. Clear differences between iDDES and $l^{2}-\omega$-DDES arise when comparing instantaneous snapshots of the flow. The appearing vortical structures of the $l^{2}-\omega$-DDES are coarser. This is caused by an increased tubulent viscosity of this approach. Moreover, this also leads to the observation that in pure LES regions, absolute values of the modeled part of any quantity are higher for the $l^{2}-\omega$-DDES. These differences arise from the varying formulations. Although the $l^{2}$ - $\omega$-DDES possesses an upper limit for the production term of the $k$-equation, the levels of modeled turbulent kinetic energy are higher than the ones of the dissipation-limited iDDES.

This behavior can also be observed in the simulations of the PennState preburner combustor. In addition to the flow field, combustion is affected as well. This yields different mixture compositions inside the chamber and thus has an impact on critical quantities such as the wall heat flux. In case of the PennState combustion chamber, the $l^{2}-\omega$-DDES clearly outperforms the iDDES as the wall heat flux of that simulation agrees better with the experimental data.

\section{Acknowledgments}

The authors gratefully acknowledge the financial support provided by the German Research Foundation (Deutsche Forschungsgemeinschaft - DFG) in the framework of the Sonderforschungsbereich Transregio 40. Computational resources have been provided by the High Performance Computing Center Stuttgart (HLRS).

\section{References}

[1] Reynolds, W. C., "The potential and limitations of direct and large eddy simulations," Whither Turbulence? Turbulence at the Crossroads, edited by J. L. Lumley, Springer Berlin Heidelberg, Berlin, Heidelberg, 1990, pp. 313-343.

[2] Chapman, D. R., “Computational aerodynamics development and outlook,” AIAA journal, Vol. 17, No. 12, 1979, pp. $1293-1313$.

[3] Spalart, P. R., Jou, W. H., Strelets, M., and Allmaras, S. R., "Comments on the feasibility of LES for wings, and on a hybrid RANS/LES approach," Advances in DNS/LES, Proceedings of the First AFOSR International Conference on DNS/LES, 1997.

[4] Strelets, M., "Detached eddy simulation of massively separated flows," 39th Aerospace Sciences Meeting and Exhibit, American Institute of Aeronautics and Astronautics, 2001. https://doi.org/10.2514/6.2001-879 


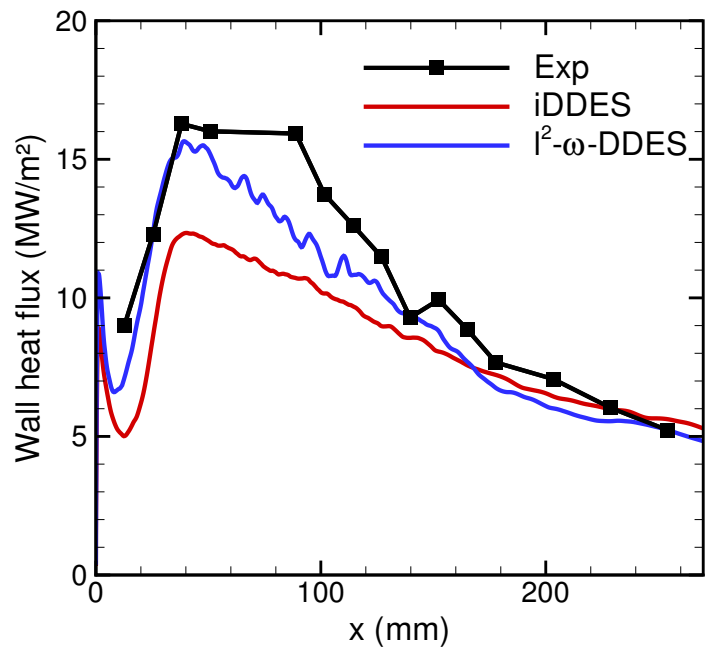

Fig. 15 Wall heat flux along the combustion chamber wall. Experimental data from [39].

[5] Spalart, P. R., Deck, S., Shur, M. L., Squires, K. D., Strelets, M. K., and Travin, A., "A new version of detached-eddy simulation, resistant to ambiguous grid densities," Theoretical and Computational Fluid Dynamics, Vol. 20, No. 3, 2006, pp. 181-195. https://doi.org/10.1007/s00162-006-0015-0

[6] Shur, M. L., Spalart, P. R., Strelets, M. K., and Travin, A. K., "A hybrid RANS-LES approach with delayed-DES and wall-modelled LES capabilities," International Journal of Heat and Fluid Flow, Vol. 29, No. 6, 2008, pp. 1638-1649. https://doi.org/10.1016/j.ijheatfluidflow.2008.07.001

[7] Reddy, K. R., Ryon, J. A., and Durbin, P. A., “A DDES model with a Smagorinsky-type eddy viscosity formulation and log-layer mismatch correction,” International Journal of Heat and Fluid Flow, Vol. 50, 2014, pp. 103-113. https: //doi.org/10.1016/j.ijheatfluidflow.2014.06.002

[8] Yin, Z., Reddy, K. R., and Durbin, P. A., "On the dynamic computation of the model constant in delayed detached eddy simulation," Physics of Fluids, Vol. 27, No. 2, 2015, p. 025105. https://doi.org/10.1063/1.4907746.

[9] Ding, P., Wang, S., and Chen, K., "Production-limited delayed detached eddy simulation of turbulent flow and heat transfer," The Canadian Journal of Chemical Engineering, Vol. 97, No. 7, 2019, pp. 2146-2156. https://doi.org/10.1002/cjce.23453

[10] Gerlinger, P., Möbus, H., and Brüggemann, D., “An implicit multigrid method for turbulent combustion,” Journal of Computational Physics, Vol. 167, No. 2, 2001, pp. 247-276.

[11] Gerlinger, P., "Multi-dimensional limiting for high-order schemes including turbulence and combustion," Journal of Computational Physics, Vol. 231, No. 5, 2012, pp. 2199-2228. https://doi.org/10.1016/j.jcp.2011.10.024

[12] Simsont, Y. H., and Gerlinger, P., "High order numerical simulation of the thermal load on a lobed strut injector for scramjet applications," International Journal for Numerical Methods in Fluids, Vol. 82, No. 7, 2016, pp. 417-436. https://doi.org/10.1002/fld.4224

[13] Shuen, J.-S., "Upwind differencing and LU factorization for chemical non-equilibrium Navier-Stokes equations," Journal of Computational Physics, Vol. 99, No. 2, 1992, pp. 233-250.

[14] Shuen, J. S., and Yoon, S., "Numerical study of chemically reacting flows using a lower-upper symmetric successive overrelaxation scheme," AIAA journal, Vol. 27, No. 12, 1989, pp. 1752-1760.

[15] Gear, C. W., Numerical initial value problems in ordinary differential equations, Prentice Hall, 1971.

[16] Liou, M.-S., “A sequel to AUSM, Part II: AUSM+-up for all speeds,” Journal of computational physics, Vol. 214, No. 1, 2006, pp. $137-170$ 
[17] Liou, M. S., "On a new class of flux splittings," Thirteenth International Conference on Numerical Methods in Fluid Dynamics, edited by M. Napolitano and F. Sabetta, Springer Berlin Heidelberg, Berlin, Heidelberg, 1993, pp. 115-119.

[18] Ó Conaire, M., Curran, H. J., Simmie, J. M., Pitz, W. J., and Westbrook, C. K., "A comprehensive modeling study of hydrogen oxidation," International journal of chemical kinetics, Vol. 36, No. 11, 2004, pp. 603-622.

[19] Gerlinger, P., "Investigation of an assumed pdf approach for finite-rate chemistry," Combustion Science and Technology, Vol. 175, No. 5, 2003, pp. 841-872. https://doi.org/10.1080/00102200302410

[20] Kim, K. H., and Kim, C., "Accurate, efficient and monotonic numerical methods for multi-dimensional compressible flows: Part II: Multi-dimensional limiting process,” Journal of computational physics, Vol. 208, No. 2, 2005, pp. 570-615.

[21] Lempke, M., Keller, R., and Gerlinger, P., "Influence of spatial discretization and unsteadiness on the simulation of rocket combustors," International Journal for Numerical Methods in Fluids, Vol. 79, No. 9, 2015, pp. 437-455. https: //doi.org/10.1002/fld.4059

[22] Kindler, M., Gerlinger, P., and Aigner, M., "Investigation of hybrid RANS/LES approaches for compressible high speed flows," 17th AIAA International Space Planes and Hypersonic Systems and Technologies Conference, 2011, p. 2218.

[23] Kindler, M., "Verbrennungssimulation in instationären kompressiblen Strömungen,” Ph.D. thesis, Universität Stuttgart, 2014.

[24] Hickey, J.-P., Ma, P. C., Ihme, M., and Thakur, S. S., "Large eddy simulation of shear coaxial rocket injector: Real fluid effects," 49th AIAA/ASME/SAE/ASEE Joint PropulsionConference, 2013, p. 4071.

[25] Wilcox, D. C., "Reassessment of the scale-determining equation for advanced turbulence models," AIAA Journal, Vol. 26, No. 11, 1988, pp. 1299-1310. https://doi.org/10.2514/3.10041.

[26] Wilcox, D. C., "Formulation of the k- $\omega$ turbulence model revisited," AIAA Journal, Vol. 46, No. 11, 2008, pp. $2823-2838$. https://doi.org/10.2514/1.36541

[27] He, C., Liu, Y., and Yavuzkurt, S., "A dynamic delayed detached-eddy simulation model for turbulent flows," Computers \& Fluids, Vol. 146, 2017, pp. 174-189. https://doi.org/10.1016/j.compfluid.2017.01.018.

[28] Comte-Bellot, G., and Corrsin, S., "Simple Eulerian time correlation of full-and narrow-band velocity signals in grid-generated, 'isotropic' turbulence,” Journal of Fluid Mechanics, Vol. 48, No. 2, 1971, pp. 273-337.

[29] Mansour, N., and Wray, A., “Decay of isotropic turbulence at low Reynolds number," Physics of Fluids, Vol. 6, No. 2, 1994, pp. $808-814$.

[30] Lee, M., and Moser, R. D., "Direct numerical simulation of turbulent channel flow up to $\operatorname{Re}_{\tau} \approx 5200$," Journal of Fluid Mechanics, Vol. 774, 2015, pp. 395-415.

[31] Davidson, L., "Large eddy simulations: how to evaluate resolution," International Journal of Heat and Fluid Flow, Vol. 30, No. 5, 2009, pp. 1016-1025.

[32] Piomelli, U., Balaras, E., Pasinato, H., Squires, K. D., and Spalart, P. R., "The inner-outer layer interface in large-eddy simulations with wall-layer models," International Journal of heat and fluid flow, Vol. 24, No. 4, 2003, pp. 538-550.

[33] Pope, S. B., Turbulent flows, Cambridge University Press, 2001.

[34] Driver, D. M., and Seegmiller, H. L., "Features of a reattaching turbulent shear layer in divergent channel flow," AIAA Journal, Vol. 23, No. 2, 1985, pp. 163-171.

[35] Driver, D. M., Seegmiller, H. L., and Marvin, J. G., "Time-dependent behavior of a reattaching shear layer," AIAA Journal, Vol. 25, No. 7, 1987, pp. 914-919.

[36] Shur, M. L., Spalart, P. R., Strelets, M. K., and Travin, A. K., "An enhanced version of DES with rapid transition from RANS to LES in separated flows," Flow, Turbulence and Combustion, Vol. 95, No. 4, 2015, pp. 709-737. https://doi.org/10.1007/s10494015-9618-0

[37] Tucker, P. K., Menon, S., Merkle, C. L., Oeflein, J. C., and Yang, V., "Validation of high-fidelity CFD simulations for rocket injector design," 44th AIAA/ASME/SAE/ASEE Joint Propulsion Conference \& Exhibit, 2008, p. 5226.

[38] Ma, P. C., Wu, H., Ihme, M., and Hickey, J.-P., "A flamelet model with heat-loss effects for predicting wall-heat transfer in rocket engines," 53rd AIAA/SAE/ASEE Joint Propulsion Conference, American Institute of Aeronautics and Astronautics, 2017. https://doi.org/10.2514/6.2017-4856 
[39] Marshall, W., Pal, S., Woodward, R., and Santoro, R., "Benchmark wall heat flux data for a GO2/GH2 single element combustor," 41st AIAA/ASME/SAE/ASEE Joint Propulsion Conference \& Exhibit, American Institute of Aeronautics and Astronautics, 2005, p. 3572. https://doi.org/10.2514/6.2005-3572

[40] Masquelet, M. M., "Large-eddy simulations of high-pressure shear coaxial flows relevant for H2/O2 rocket engines," $\mathrm{Ph} . \mathrm{D}$. thesis, Georgia Institute of Technology, 2013.

[41] Tucker, P., Menon, S., Merkle, C., Oefelein, J., and Yang, V., "An approach to improved credibility of CFD calculations for rocket injector design," 43rd AIAA/ASME/SAE/ASEE Joint Propulsion Conference \& Exhibit, American Institute of Aeronautics and Astronautics, 2007, p. 5572. https://doi.org/10.2514/6.2007-5572 